\title{
A local formulation of lattice QCD without unphysical fermion zero modest
}

\author{
Roberto Frezzotti ${ }^{\mathrm{a}}$, Pietro Antonio Grassi ${ }^{\mathrm{a}}$, Stefan Sint ${ }^{\mathrm{b}}$ and Peter Weisz ${ }^{\mathrm{a}}$ \\ àMax-Planck-Institut für Physik, Föhringer Ring 6, D-80805 München, Germany \\ bUniversità di Roma "Tor Vergata", Dipartimento di Fisica, Via della Ricerca Scientifica 1, I-00133 \\ Roma, Italy
}

The problem of unphysical zero modes in lattice QCD with Wilson fermions can be solved in a clean way by including a mass term proportional to $i \bar{\psi} \gamma_{5} \tau^{3} \psi$ in the standard lattice theory with $N_{\mathrm{f}}=2$ mass degenerate Wilson quarks. We argue that up to cutoff effects, this lattice theory is equivalent to standard lattice QCD, for suitable choices of the mass parameters and with a natural re-interpretation of observables. On-shell $\mathrm{O}(a)$ improvement can be implemented in a straightforward way.

\section{INTRODUCTION}

Lattice QCD with Wilson quarks provides an attractive framework for non-perturbative computations of many interesting phenomenological quantities. Its main disadvantage consists in the explicit breaking of all axial symmetries. As a consequence the Wilson-Dirac operator is not protected against zero modes even at finite values of the quark mass. While this is not a theoretical problem for the full theory, it severely limits the applicability of the quenched approximation as the occurrence of unusually small eigenvalues may lead to large fluctuations in observable quantities. In practice this means that one cannot directly treat the light quark masses but has to extrapolate results obtained with relatively heavy quarks. It is important to appreciate that this limitation is completely independent of the usual requirement of not too light pion masses, dictated by the finite space-time volume used for the simulation. Indeed quite often quenched simulations are limited by the occurrence of "exceptional configurations" at quark mass values where finite volume effects are still completely negligible.

Various recipes have been proposed to deal with this problem [1-3] but to our knowledge no

\footnotetext{
*based on a talk by R. Frezzotti and a poster by S. Sint, presented at the International Symposium on Lattice Field Theory, June 29 - July 3, 1999, Pisa, Italy
}

completely satisfactory solution has appeared in the literature. In order to fill this gap, we consider the lattice action first introduced by Aoki [4. More precisely, if $D_{\mathrm{W}}$ denotes the $(\mathrm{O}(a)$ improved) Wilson-Dirac operator for a quark with bare mass $m_{0}$, we consider the fermionic action for $N_{\mathrm{f}}=2$ quarks with the lattice Dirac operator

$D_{\text {twist }} \stackrel{\text { def }}{=} D_{\mathrm{W}}+i \mu_{\mathrm{q}} \gamma_{5} \tau^{3}$.

The additional term has a non-trivial flavour structure (the Pauli matrix $\tau^{3}$ acts in flavour space), and will be referred to as a chirally twisted mass term. It is obvious that this lattice Dirac operator is protected against zero modes for any finite value of $\mu_{\mathrm{q}}$,

$\operatorname{det} D_{\text {twist }}=\operatorname{det}\left(D_{\mathrm{W}}^{\dagger} D_{\mathrm{W}}+\mu_{\mathrm{q}}^{2}\right)>0$.

To use this lattice action as a regularization of "exceptional configurations" has therefore been proposed in ref. [5], implying, however, that the mass parameter $\mu_{\mathrm{q}}$ be extrapolated to zero at the end. As will become clear below, this is not necessary. It can be shown that lattice QCD with a chirally twisted mass term (twisted lattice QCD) is, up to cutoff effects, completely equivalent to standard lattice QCD, and thus provides an alternative regularization of the massive theory.

This article is organised as follows: we first take a closer look at the classical continuum limit of 
twisted lattice QCD (sect. 2). Assuming universality we sketch an elementary proof that twisted and standard lattice QCD are equivalent in the continuum limit (sect. 3). In sect. 4 we determine the possible counterterms, and briefly introduce our choice of renormalization scheme. We then discuss $\mathrm{O}(a)$ improvement (sect. 5) and we end with a few comments on further applications.

\section{CLASSICAL CONTINUUM THEORY}

To get acquainted with twisted QCD it is instructive to have a closer look at the corresponding classical continuum theory, described by the lagrangian

$\mathcal{L}_{f}(x)=\bar{\psi}(x)\left(\not D+m+i \mu_{\mathrm{q}} \gamma_{5} \tau^{3}\right) \psi(x)$.

Defining the usual isospin currents and densities,

$A_{\mu}^{a}=\bar{\psi} \gamma_{\mu} \gamma_{5} \frac{\tau^{a}}{2} \psi$,

$V_{\mu}^{a}=\bar{\psi} \gamma_{\mu} \frac{\tau^{a}}{2} \psi$,

$P^{a}=\bar{\psi} \gamma_{5} \frac{\tau^{a}}{2} \psi$,

the partial gonservation of axial and vector currents reads

$\partial_{\mu} A_{\mu}^{a}=2 m P^{a}, \quad a=1,2$

$\partial_{\mu} A_{\mu}^{3}=2 m P^{3}+i \mu_{\mathrm{q}} \bar{\psi} \psi$,

$\partial_{\mu} V_{\mu}^{a}=-2 \mu_{\mathrm{q}} \varepsilon_{a b} P^{b}, \quad a, b=1,2$

$\partial_{\mu} V_{\mu}^{3}=0$.

A global chiral (non-singlet) rotation of the fields,

$\psi^{\prime}=\exp \left(i \alpha \gamma_{5} \tau^{3} / 2\right) \psi$,

$\bar{\psi}^{\prime}=\bar{\psi} \exp \left(i \alpha \gamma_{5} \tau^{3} / 2\right)$,

with $\tan \alpha=\mu_{\mathrm{q}} / m_{\mathrm{q}}$ transforms the Lagrangian to its standard form,

$\mathcal{L}_{f}^{\prime}(x)=\bar{\psi}^{\prime}(x)\left(\not D+m^{\prime}\right) \psi^{\prime}(x)$,

with the quark mass $m^{\prime}=\sqrt{m^{2}+\mu_{\mathrm{q}}^{2}}$. The isospin currents and densities in the primed basis

\footnotetext{
${ }^{2}$ We define the antisymmetric tensor $\varepsilon_{a b}$ with indices $a, b=1,2$ and normalization $\varepsilon_{12}=1$. Summation over repeated indices is always understood.
}

are related to the original fields through $(a, b=$ $1,2)$

${A^{\prime}}_{\mu}^{a}=\cos (\alpha) A_{\mu}^{a}+\sin (\alpha) \varepsilon_{a b} V_{\mu}^{b}$,

$A^{\prime 3}=A_{\mu}^{3}$,

$V_{\mu}^{\prime a}=\cos (\alpha) V_{\mu}^{a}+\sin (\alpha) \varepsilon_{a b} A_{\mu}^{b}$,

$V_{\mu}^{\prime 3}=V_{\mu}^{3}$

$P^{\prime a}=P^{a}$,

$P^{\prime 3}=\cos (\alpha) P^{3}+i \sin (\alpha) \frac{1}{2} \bar{\psi} \psi$,

and satisfy the PCAC and PCVC relations in their standard forms, i.e.

$\partial_{\mu} A^{\prime a}=2 m^{\prime} P^{\prime a}, \quad \partial_{\mu} V_{\mu}^{\prime a}=0, \quad a=1,2,3$.

Similar relationships can be worked out between more complicated composite fields, and we thus conclude that the classical twisted theory can be mapped back to the standard formulation by a simple axial rotation of the fields, with a unique rotation angle which is fixed by the ratio of the mass parameters. Both formulations are thus equivalent and contain the same physical information.

\section{BEYOND THE CLASSICAL THEORY}

In order to establish the equivalence between twisted QCD and the standard formulation of QCD beyond the classical continuum limit, it is convenient to make use of a regularization which does not break the flavour symmetries. This is possible [6, [7] if the lattice Dirac operator satisfies the Ginsparg-Wilson relation [8],

$D \gamma_{5}+\gamma_{5} D=a D \gamma_{5} D$.

An explicit solution for $D$ has been given by Neuberger [9], but in the following we only need to know that $D$ is a local operator which satisfies the Ginsparg-Wilson relation and has the conjugation property $D^{\dagger}=\gamma_{5} D \gamma_{5}$. It then follows that the matrix [10],

$\hat{\gamma}_{5} \stackrel{\text { def }}{=} \gamma_{5}(1-a D)$,

is hermitian and unitary. Therefore it may be used to define left handed quark fields through

$\psi_{\mathrm{L}}=\frac{1}{2}\left(1-\hat{\gamma}_{5}\right) \psi, \quad \bar{\psi}_{\mathrm{L}}=\bar{\psi} \frac{1}{2}\left(1+\gamma_{5}\right)$, 
and right handed fields are obtained with the complementary projectors. Using these chiral fields a lattice regularized version of twisted QCD is specified by the action

$$
\begin{aligned}
S_{f}=a^{4} \sum_{x}[ & \bar{\psi}_{\mathrm{L}} D \psi_{\mathrm{L}}+\bar{\psi}_{\mathrm{R}} D \psi_{\mathrm{R}} \\
& +m\left(\bar{\psi}_{\mathrm{L}} \psi_{\mathrm{R}}+\bar{\psi}_{\mathrm{R}} \psi_{\mathrm{L}}\right) \\
& \left.+i \mu_{\mathrm{q}}\left(\bar{\psi}_{\mathrm{L}} \tau^{3} \psi_{\mathrm{R}}-\bar{\psi}_{\mathrm{R}} \tau^{3} \psi_{\mathrm{L}}\right)\right] .
\end{aligned}
$$

In the chiral limit $m=\mu_{\mathrm{q}}=0$ this action has an exact $\mathrm{SU}(2) \times \mathrm{SU}(2)$ flavour symmetry, and in particular the axial transformation [cf. eq. (11)],

$$
\begin{aligned}
\psi_{\mathrm{L}}^{\prime} & =\exp \left(-i \alpha \tau^{3} / 2\right) \psi_{\mathrm{L}}, \\
\bar{\psi}_{\mathrm{L}}^{\prime} & =\bar{\psi}_{\mathrm{L}} \exp \left(i \alpha \tau^{3} / 2\right), \\
\psi_{\mathrm{R}}^{\prime} & =\exp \left(i \alpha \tau^{3} / 2\right) \psi_{\mathrm{R}}, \\
\bar{\psi}_{\mathrm{R}}^{\prime} & =\bar{\psi}_{\mathrm{R}} \exp \left(-i \alpha \tau^{3} / 2\right),
\end{aligned}
$$

is part of the symmetry group. Therefore, this transformation leaves the form of the lattice action invariant and merely transforms the parameters,

$$
\begin{aligned}
m^{\prime} & =m \cos (\alpha)+\mu_{\mathrm{q}} \sin (\alpha), \\
\mu_{\mathrm{q}}^{\prime} & =-m \sin (\alpha)+\mu_{\mathrm{q}} \cos (\alpha),
\end{aligned}
$$

where the choice $\tan \alpha=\mu_{\mathrm{q}} / m$ again produces the standard action with $\mu_{\mathrm{q}}^{\prime}=0$ and $m^{\prime}=$ $\left(m^{2}+\mu_{\mathrm{q}}^{2}\right)^{1 / 2}$. Since the flavour symmetry in the basis of chiral fields takes exactly the same form as in the continuum, we may also reproduce eqs. (13-19). More generally, bare operators in a given representation of the flavour symmetry group are rotated covariantly by the same angle $\alpha$. Finally we note that the measure of the functional integral is left invariant under the axial (non-singlet) transformation, eq. 24). We have thus found a regularization where (at least in a finite space-time volume) the bare parameters and correlation functions of twisted QCD are related to the ones of standard QCD through a change of variables in a well-defined functional integral. This yields exact identities at any fixed value of the lattice spacing, and gives a precise meaning to the equivalence between the two theories. For example, if $O_{k}$ and $O_{k}^{\prime}$, are, for $k=1, \ldots, n$, bare gauge invariant composite operators, which are related through the axial transformation (24), we obtain the identity between the (bare) connected correlation functions,

$$
\begin{aligned}
& \left\langle O_{1}\left(x_{1}\right) \cdots O_{n}\left(x_{n}\right)\right\rangle_{c}^{\text {twisted QCD }}= \\
& \left\langle O_{1}^{\prime}\left(x_{1}\right) \cdots O_{n}^{\prime}\left(x_{n}\right)\right\rangle_{c},
\end{aligned}
$$

provided the bare parameters $\mu_{\mathrm{q}}, m$ and $m^{\prime}$ are related as indicated previously.

In order for these relations to carry over to the continuum limit, it is convenient to renormalize both mass parameters, $\mu_{\mathrm{q}}$ and $m$ in the same way, such that the ratio $\mu_{\mathrm{q}} / m$, and thus the angle $\alpha$, remains unchanged. If the chosen renormalization scheme respects the global flavour symmetries, then the relations between correlation functions of composite operators are exactly the same as in the bare theory, and thus also hold in the continuum limit. Mass independent renormalization schemes obviously have this property and in addition preserve relations between renormalized parameters, but it turns out that a much wider class of renormalization schemes is admissible 11].

In lattice QCD with Wilson fermions an axial transformation does not correspond to an exact symmetry. However, assuming universality of the continuum limit, we may conclude that the twisted and standard formulations of lattice QCD with Wilson fermions are indeed equivalent up to cutoff effects. In fact, this can be proven rigorously to all orders in the loop expansion, by exploiting reparametrization invariance and the Quantum Action Principle 11]. In particular, this proof holds for any regularization for which a power counting theorem can be established, regardless of whether or not the flavour symmetries are broken by the regularization.

\section{RENORMALIZATION}

On the lattice with Wilson fermions only the isospin $\mathrm{SU}(2)$ symmetry remains exact, and the flavour symmetry is further reduced by the introduction of the twisted mass term. This is in contrast with the lattice regularization based on Ginsparg-Wilson fermions, where the symmetries are exactly the same in both (standard and 
twisted) formulations, albeit realized in a different way.

A careful analysis shows that, apart from gauge invariance and invariance under discrete fourdimensional rotations, the following symmetries remain exact on the lattice:

- a global U(1) symmetry corresponding to fermion number conservation,

- a residual U(1) flavour symmetry with generator $\tau^{3} / 2$,

- charge conjugation,

- $P_{F}$ : parity combined with flavour exchange,

- $\tilde{F}$ : flavour exchange combined with $\mu_{\mathrm{q}} \rightarrow$ $-\mu_{\mathrm{q}}$.

Furthermore, it is possible to show that reflection positivity 12 still holds.

Taking into account these symmetries, twisted lattice QCD is renormalizable by power counting. The only new counterterm of dimension $\leq 4$ is proportional to $i \mu_{\mathrm{q}} \bar{\psi} \gamma_{5} \tau^{3} \psi$, which leads to a multiplicative renormalization of $\mu_{\mathrm{q}}$. In particular we note that a term of the form $\varepsilon_{\mu \nu \rho \sigma} \operatorname{tr}\left\{F_{\mu \nu} F_{\rho \sigma}\right\}$ is excluded by the symmetry $P_{F}$, whereas invariance under $\tilde{F}$ excludes an additive renormalization of $\mu_{\mathrm{q}}$ (i.e. a counterterm proportional to $\left.a^{-1} i \bar{\psi} \gamma_{5} \tau^{3} \psi\right)$.

We would like to impose renormalization conditions such that the relation between renormalized lattice QCD and its twisted version is as simple as in the classical continuum limit. However, due to the reduced flavour symmetry with Wilson fermions some preparation is needed. In particular, one has to determine the relative normalization between composite operators that belong to the same representation of the continuum flavour symmetry, but are not related by lattice symmetries. This can be achieved by imposing continuum chiral Ward identities as normalization conditions for the lattice operators [13]. An example is provided by the isospin currents: while the conserved vector current is protected against renormalization, the correctly normalized axial current $\left(A_{\mathrm{R}}\right)_{\mu}^{a}$ has to be determined by imposing current algebra relations as normalization conditions 14.
We then expect that relations like eqs.(13-18) hold for the correctly normalized currents, provided the angle $\alpha$ is defined using the Ward identity masses. More precisely, we define a bare current quark mass $m$ through (some suitable matrix element of) the PCAC relation,

$\partial_{\mu}\left(A_{\mathrm{R}}\right)_{\mu}^{1}=2 m P^{1}$,

and define $\alpha$ through

$\tan \alpha=\mu_{\mathrm{q}} / m$.

Note that $m$ differs from the subtracted bare quark mass $m_{\mathrm{q}}=m_{0}-m_{\mathrm{c}}$. In the renormalized theory, we thus fix the ratio of the mass renormalization constants

$m_{\mathrm{R}}=Z_{\mathrm{m}} m_{\mathrm{q}}, \quad \mu_{\mathrm{R}}=Z_{\mu} \mu_{\mathrm{q}}$,

such that it coincides with the slope at the origin of the function $m\left(m_{\mathrm{q}}\right)$.

\section{O(a) IMPROVEMENT}

Given the symmetries of the lattice theory it is a straightforward exercise to list the possible $\mathrm{O}(a)$ counterterms to the action. The procedure has been explained in great detail in ref. [15] and applies to the case at hand, taking into account the modified set of symmetries given in the previous section. We expect the renormalization and $\mathrm{O}(a)$ improvement of the massless theory to be the same as in standard lattice QCD [13-15]. This follows from analyticity of correlation functions in the mass parameters $m_{\mathrm{q}}$ and $\mu_{\mathrm{q}}$, which is however guaranteed only with an infrared cutoff, e.g. in a finite space-time volume.

With this assumption, on-shell $\mathrm{O}(a)$ improvement of the action is achieved by using the Sheikholeslami-Wohlert improved Wilson-Dirac operator [16], and the following parameterization of the $\mathrm{O}(a)$ improved renormalized coupling and quark masses,

$$
\begin{aligned}
g_{\mathrm{R}}^{2} & =\tilde{g}_{0}^{2} Z_{\mathrm{g}}\left(\tilde{g}_{0}^{2}, a \mu\right), \\
m_{\mathrm{R}} & =\widetilde{m}_{\mathrm{q}} Z_{\mathrm{m}}\left(\tilde{g}_{0}^{2}, a \mu\right), \\
\mu_{\mathrm{R}} & =\widetilde{\mu}_{\mathrm{q}} Z_{\mu}\left(\tilde{g}_{0}^{2}, a \mu\right), \\
\tilde{g}_{0}^{2} & =g_{0}^{2}\left(1+b_{\mathrm{g}} a m_{\mathrm{q}}\right), \\
\tilde{m}_{\mathrm{q}} & =m_{\mathrm{q}}+b_{\mathrm{m}} a m_{\mathrm{q}}^{2}+\tilde{b}_{\mathrm{m}} a \mu_{\mathrm{q}}^{2},
\end{aligned}
$$


$\widetilde{\mu}_{\mathrm{q}}=\mu_{\mathrm{q}}\left(1+b_{\mu} a m_{\mathrm{q}}\right)$

$m_{\mathrm{q}}=m_{0}-m_{\mathrm{c}}$.

This notation already implies our choice of a mass-independent renormalization scheme 18 , 19. $\mathrm{O}(a)$ improvement of the action thus introduces the improvement coefficients $b_{\mu}$ and $\tilde{b}_{\mathrm{m}}$, in addition to the standard coefficients $b_{\mathrm{m}}$ and $b_{\mathrm{g}}$.

As for the improvement of operators we here give a few examples which appear in the PCAC and PCVC relations. Following ref. 15] we define the improved operators $(a=1,2)$,

$$
\begin{aligned}
\left(A_{\mathrm{I}}\right)_{\mu}^{a} & =A_{\mu}^{a}+c_{\mathrm{A}} a \tilde{\partial}_{\mu} P^{a}+a \mu_{\mathrm{q}} \tilde{b}_{\mathrm{A}} \varepsilon_{a b} V_{\mu}^{b} \\
\left(V_{\mathrm{I}}\right)_{\mu}^{a} & =V_{\mu}^{a}+c_{\mathrm{V}} a \tilde{\partial}_{\nu} T_{\mu \nu}^{a}+a \mu_{\mathrm{q}} \tilde{b}_{\mathrm{V}} \varepsilon_{a b} A_{\mu}^{b} \\
\left(P_{\mathrm{I}}\right)^{a} & =P^{a}
\end{aligned}
$$

and obtain the renormalized improved operators through $(a=1,2)$ :

$$
\begin{aligned}
\left(A_{\mathrm{R}}\right)_{\mu}^{a} & =Z_{\mathrm{A}}\left(1+b_{\mathrm{A}} a m_{\mathrm{q}}\right)\left(A_{\mathrm{I}}\right)_{\mu}^{a}, \\
\left(V_{\mathrm{R}}\right)_{\mu}^{a} & =Z_{\mathrm{V}}\left(1+b_{\mathrm{V}} a m_{\mathrm{q}}\right)\left(V_{\mathrm{I}}\right)_{\mu}^{a}, \\
\left(P_{\mathrm{R}}\right)^{a} & =Z_{\mathrm{P}}\left(1+b_{\mathrm{P}} a m_{\mathrm{q}}\right)\left(P_{\mathrm{I}}\right)^{a} .
\end{aligned}
$$

Note that we may avoid technical problems associated with power divergences by restricting attention to the first two isospin components of these operators. This can be done without any loss of information due to the expected restoration of all chiral Ward identities in the continuum limit.

With these definitions the $\mathrm{O}(a)$ improved angle $\alpha$ is defined by

$\tan \alpha=\frac{\mu_{\mathrm{R}}}{m_{\mathrm{R}}}=\frac{Z_{\mu} \widetilde{\mu}_{\mathrm{q}}}{Z_{\mathrm{m}} \widetilde{m}_{\mathrm{q}}}$

where $\mu_{\mathrm{R}}$ and $m_{\mathrm{R}}$ are obtained from matrix elements of the renormalized $\mathrm{O}(a)$ improved PCAC and $\mathrm{PCVC}$ relations. Again this requires that the ratio $Z_{\mu} / Z_{\mathrm{m}}$ be fixed as explained in the previous section.

In order to check the general framework of renormalization and $\mathrm{O}(a)$ improvement described above, we carried out a perturbative calculation using on-shell correlation functions derived from the Schrödinger functional 20]. The details of the calculations are very similar to the studies of the standard theory [21,22] and will be described elsewhere [11]. As a result we confirm the theoretical expectations. In particular, all improvement coefficients are found to be functions of the bare coupling alone, and do not depend e.g. on the angle $\alpha$. To lowest order we find $\tilde{b}_{\mathrm{A}}^{(0)}=\tilde{b}_{\mathrm{V}}^{(0)}=b_{\mu}^{(0)}=0$ and $\tilde{b}_{m}^{(0)}=-\frac{1}{2}$, and the corresponding one-loop coefficients are available, too [11].

\section{CONCLUSIONS AND OUTLOOK}

There remains little doubt that twisted lattice QCD and standard lattice QCD for $N_{\mathrm{f}}=2$ mass degenerate quarks are equivalent theories in the continuum limit, up to $\mathrm{O}\left(a^{2}\right)$ effects if $\mathrm{O}(a)$ improvement is implemented. Since twisted lattice QCD is protected against unphysical fermion zero modes, the problem of exceptional configurations has thus found a clean solution. Furthermore, realistic studies of QCD with $N_{\mathrm{f}}>2$ can be easily done by using the (improved) standard lattice action for the heavier quarks.

In full QCD the problem of exceptional configurations is absent in principle. However, it has been argued that close to the chiral limit, standard algorithms may still experience technical problems, showing up e.g. as a poor sampling of configuration space [23,24]. Twisted QCD may therefore be useful in this context, too. Incidentally, full QCD simulations using (unimproved) twisted lattice QCD have already been carried out, albeit with a different motivation 25].

In view of $\mathrm{O}(a)$ improvement, there are preferred choices of $\alpha$. If $\alpha$ is small, perturbative estimates of the new $b$-coefficients are sufficient, while large fluctuations due to close zero modes are already excluded. Another attractive possibility is the choice $\alpha=\pi / 2$, such that the physical quark mass is entirely defined in terms of $\mu_{R}$. In this case all the standard $b$-coefficients are not needed, and, in particular, $\tilde{g}_{0}=g_{0}$. This latter fact may be interesting for full QCD simulations, as continuum and chiral extrapolations may become easier.

Since theories with different values of $\alpha$ are not related by a lattice symmetry, operator mixing problems may look quite different. A simple example is provided by the iso-singlet scalar density where the usual additive renormalization at $\alpha=0$ can be avoided by working at $\alpha=\pi / 2$. 
Dynamical fermion simulations being very expensive it has been proposed to obtain information about full QCD by extrapolating from the unphysical region where $N_{\mathrm{f}} \leq 0$ [26-28]. For this "bermion" approach the problem of close zero modes is much more severe than in the quenched approximation, and may again be solved using twisted lattice QCD.

Originally, Aoki introduced the chirally twisted mass term to discuss the phase structure of the standard lattice theory [4]. At strong coupling he established the existence of a phase with spontaneous breaking of parity. We do not exclude that such an Aoki phase also exists at weaker couplings. However, even if it does, this would be relevant only in large space-time volume and very close to the chiral limit (mass parameters of $\mathrm{O}\left(a^{2}\right)$ ) where simulations are not practical anyway (cf. ref. 29]).

In conclusion, we believe that twisted lattice QCD provides a very promising alternative formulation of lattice QCD.

This work is part of the ALPHA collaboration research programme. We are grateful to M. Lüscher for useful suggestions, and to R. Sommer for a critical reading of our notes. S. Sint acknowledges support by the European Commission under grant No. FMBICT972442.

\section{REFERENCES}

[1] W. Bardeen et al., Phys. Rev. D57 (1998) 1633

[2] A. Hoferichter et al., Nucl. Phys. B (Proc. Suppl.) 63 (1998) 164

[3] M. Göckeler et al., Nucl. Phys. B (Proc. Suppl.) 73 (1999) 889

[4] S. Aoki, Phys. Rev. D30 (1984) 2653

[5] W. Bardeen, A. Duncan, E. Eichten and H. Thacker, Phys. Rev. D59 (1999) 014507

[6] P. Hasenfratz, Nucl. Phys. B525 (1998) 401

[7] M. Lüscher, Phys. Lett. B428 (1998) 342

[8] P.H. Ginsparg and K.G. Wilson, Phys. Rev. D25 (1982) 2649

[9] H. Neuberger, Phys. Lett. B417 (1998) 141

[10] M. Lüscher, Nucl. Phys. B549 (1999) 295

[11]R. Frezzotti, P.A. Grassi, S. Sint and P. Weisz, work in progress
[12] K. Osterwalder and E. Seiler, Ann. of Phys. 110 (1978) 440; G. Immirzi and K. Yoshida, Nucl. Phys. B210[FS6] (1982) 499 and references therein

[13] M. Bochicchio et al., Nucl. Phys. B262 (1985) 331

[14] M. Lüscher, S. Sint, R. Sommer and H. Wittig, Nucl. Phys. B491 (1997) 344

[15] M. Lüscher, S. Sint, R. Sommer and P. Weisz, Nucl. Phys. B478 (1996) 365

[16] B. Sheikholeslami and R. Wohlert, Nucl. Phys. B259 (1985) 572

[17] M. Lüscher et al., Nucl. Phys. B491 (1997) 323

[18] S. Weinberg, Phys. Rev. D8 (1973) 3497

[19] K. Jansen et al., Phys. Lett. B372 (1996) 275

[20] S. Sint, Nucl. Phys. B421 (1994) 135; Nucl. Phys. B451 (1995) 416

[21] M. Lüscher and P. Weisz, Nucl. Phys. B479 (1996) 429

[22] S. Sint and P. Weisz, Nucl. Phys. B502 (1997) 251; Nucl. Phys. B (Proc. Suppl.) 63 (1998) 856

[23] K. Jansen and R. Sommer, Nucl. Phys. B530 (1998) 185

[24]R. Frezzotti and K. Jansen, Phys. Lett. B402 (1997) 328; hep-lat/9808011; heplat $/ 9808038$

[25] K.M. Bitar, Phys. Rev. D56 (1997) 2736; Nucl. Phys. B (Proc. Suppl.) 63 (1998) 829

[26] S.J. Anthony, C.H. Llewellyn Smith and J.F. Wheather, Phys. Lett. 116B (1982) 287

[27] G.M. de Divitiis et al., Nucl. Phys. B455 (1995) 274 and references therein

[28] R. Petronzio, Nucl. Phys. B (Proc. Suppl.) 42 (1995) 942

[29] S. Sharpe and R. Singleton, Phys. Rev. D58 (1998) 074501 